\title{
Collaborative supply chain planning - A case study from the German cutting tool industry
}

\author{
Gregor von Cieminski, Carsten Begemann, Stefan Lutz, Michael Schneider and \\ Hans-Peter Wiendahl \\ Institute of Production Systems and Logistics (IFA), University of Hanover, Callinstrasse 36, \\ D-30167 Hannover, Germany.Email: cieminski@ifa.uni-hannover.de
}

\begin{abstract}
This paper is concerned with the case study of the supply chain of a German cutting tool manufacturer. The Institute of Production Systems and Logistics (IFA) carried out a comprehensive analysis of the supply chain in cooperation with the companies that are partners in the chain. The analysis was carried out from a production logistics perspective. By use of the theory of logistic operating curves, logistic performance measures for manufacturing departments as well as for product stores were determined and potentials for improvement identified. The supply chain tiers were initially considered separately. This facilitated a detailed analysis of their specific logistic performance and its causes. Merging the results of the investigations led to a thorough understanding of the interdependencies that exist between the performances of different parts of a supply chain. The results of the individual studies were consolidated in order to support a more holistic approach to production management and to better achieve the objectives of the entire chain. A transparent combined Kanban-CONWIP control cycle was suggested in order to improve the collaborative production planning and control of the complete supply chain.
\end{abstract}

Keywords

Supply chain management, industrial case study, logistic performance measures, logistic operating curves.

\section{SUPPLY CHAIN MANAGEMENT}

Definitions of Supply Chain Management (SCM) emphasise the collaborative aspects that this management paradigm incorporates - on a strategic (Schönsleben, 2000) as well as on an operational level (Wiendahl et al., 1998). Hieber and Alard (1999) state that there is still a gap between the conceptual ideas of supply chain management and its application in industry. This is especially true for the case of small to medium-sized enterprises (SMEs). The paper presents a case study of a medium-sized German cutting tool manufacturer that reinforces this impression. The supply chain of this company was analysed in a comprehensive logistic investigation. This revealed the disparities between the best practices of SCM and 
their implementation in practice: companies in supply chains pursue individual rather than common objectives.

The analysis carried out concentrated on the supply chain tiers shown in Figure 1. This section of the supply chain consists of two companies: the cutting tool manufacturer and its major supplier of semi-finished products. The semi-finished products store and the final products store constitute two further tiers of the section.

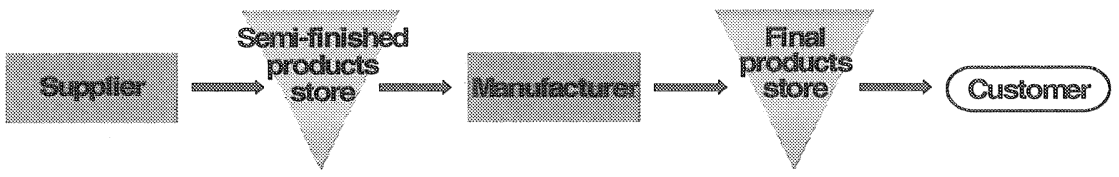

Figure 1 - Supply chain of cutting tool manufacturer (section considered)

The supplier employs a sequence of 5 different processes to produce the semifinished products. These are delivered daily to the semi-finished products store at the manufacturer's site. The manufacturer applies a further 3 processes to complete the final products. The final products are manufactured to stock so that forecast customer demands can be satisfied from the final products store. The two companies have a trusting, cooperative working relationship, which was further underpinned by their willingness to actively engage in the studies undertaken by an external consultant. However, despite the fact that their cooperation went as far as sharing the PPC system, dedicated SCM practices had not been put into effect. The companies continued to pursue their own economic as well as logistic objectives, regardless of the problems they were thus creating for each other. The main problem that was cited at the outset of the investigations was that the supply chain had difficulties in guaranteeing a satisfactory availability of the final products to its customers. Long throughput times across the whole chain were deemed to be a significant cause of this. At the same time the WIP and inventory levels of the supply chain were relatively high. This seemed to run contrary to the principle of a smooth material flow through a lean production set up.

In order to be able to better explain the procedure and objectives of the logistic analysis methods, the paper firstly presents the different tools applied for analysing separate tiers of the supply chain. The results of the assessment of the supply chain tiers are also provided in this section. Later Section consolidates these results from a holistic view of the supply chain performance. By identifying the interdependencies of the performances of different supply chain tiers, suitable mechanisms for a more collaborative supply chain planning can be recommended.

\section{ANALYSES OF SUPPLY CHAIN TIERS}

In a series of four logistics analyses, the Institute of Production Systems and Logistics (IFA) studied the four tiers of the supply chain shown in Figure 1. These analyses applied a range of models and methodologies that IFA developed specifically in order to assess and improve the logistic performance of production processes. The theoretical models utilised in the analyses of the supply chain are 
throughput diagrams for work systems (Wiendahl, 1995) and inventories (Lutz et al., 2002), logistic operating curves (Nyhuis, 1994) and inventory operating curves (Lutz et al., 2002). These models are embedded in two types of analysis methods, the bottleneck-oriented logistics analysis (Penz \& Nyhuis, 1995) and the logisticsoriented inventory analysis (Lutz et al., 2002). Two bottleneck-oriented logistics analyses were carried out for the production departments of both, manufacturer and supplier. The semi-finished products store and the final products store of the manufacturer were analysed in two logistics-oriented inventory analyses. The two analysis methods are directed at pinpointing the logistic shortcomings of the objects under study, deriving their potentials for improvement and proposing the appropriate measures to exploit these potentials. The initial analyses considered the distinct supply chain tiers as closed systems. Thus, they aspired to a local optimisation of the logistic performance measures. This section therefore describes how the analysis methods were applied in the individual studies of the four supply chain tiers and presents the results of these.

\section{Final Products Store}

The final products store is operated as a normal inventory-holding store. The manufacturer aims to satisfy customer demands for standard products immediately on request and demands for customised products with a lead time of six weeks. Internally, a performance objective of $95 \%$ customer service level has been set. The inventory levels required to anticipate customer demands are determined by exponential smoothing forecasts that form the basis of the production programme. IFA applied the logistics-oriented inventory analysis to evaluate the logistic performance of the store.

\section{Logisticsmoriented Inventory Analysis}

The detailed procedure of the logistics-oriented inventory analysis is shown in Figure 2 (Lutz et al., 2002). The logistic performance measures primarily evaluated are the mean inventory level, the customer service level and the delivery delay. Other measures considered are input and output rates and the stock turnaround. The inventory analysis has two objectives. Firstly, it aims to determine the adequate safety-stock level for every article in store by determining measures of the three influencing factors - variations in customer demands, delivery quantity deviations and delivery date deviations. Secondly, the inventory operating curves are used to determine the potentials for reduction of the current inventory levels to those target levels that guarantee the customer service level and delivery delay figures required. As shown in Figure 2, the inventory analysis is split into three phases. The preparation phase defines the scope of the analysis. The analysis phase determines all the relevant numerical values of both, performance measures and potentials. The final phase is concerned with the formulation of measures to be undertaken in order to exploit the potentials identified in the second phase. 

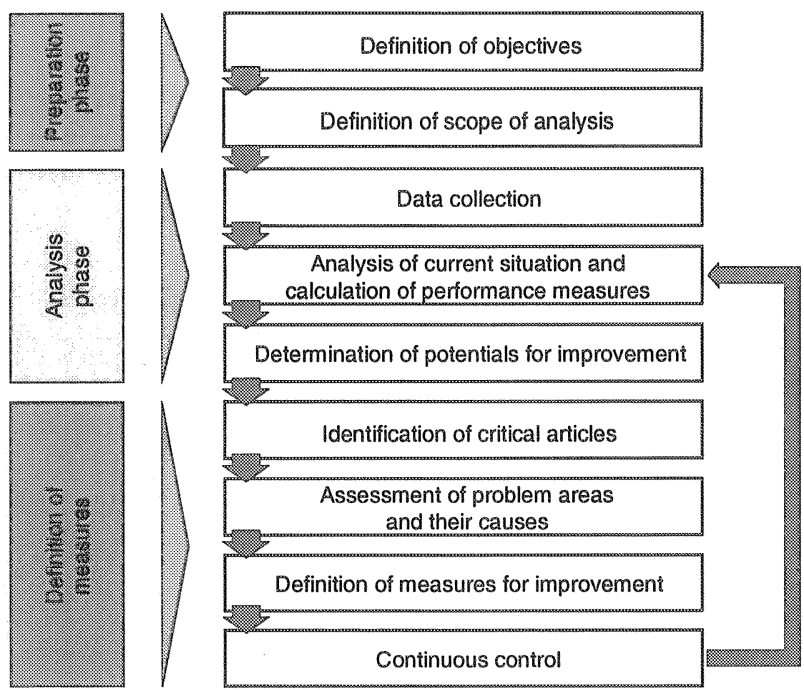

Figure 2 - Procedure of the logistics-oriented inventory analysis

\section{Inventory Operating Curves}

Figure 3 shows the inventory operating curves of a sample article: customer service level and delivery delay are functions of the mean inventory level (Lutz et al., 2002).
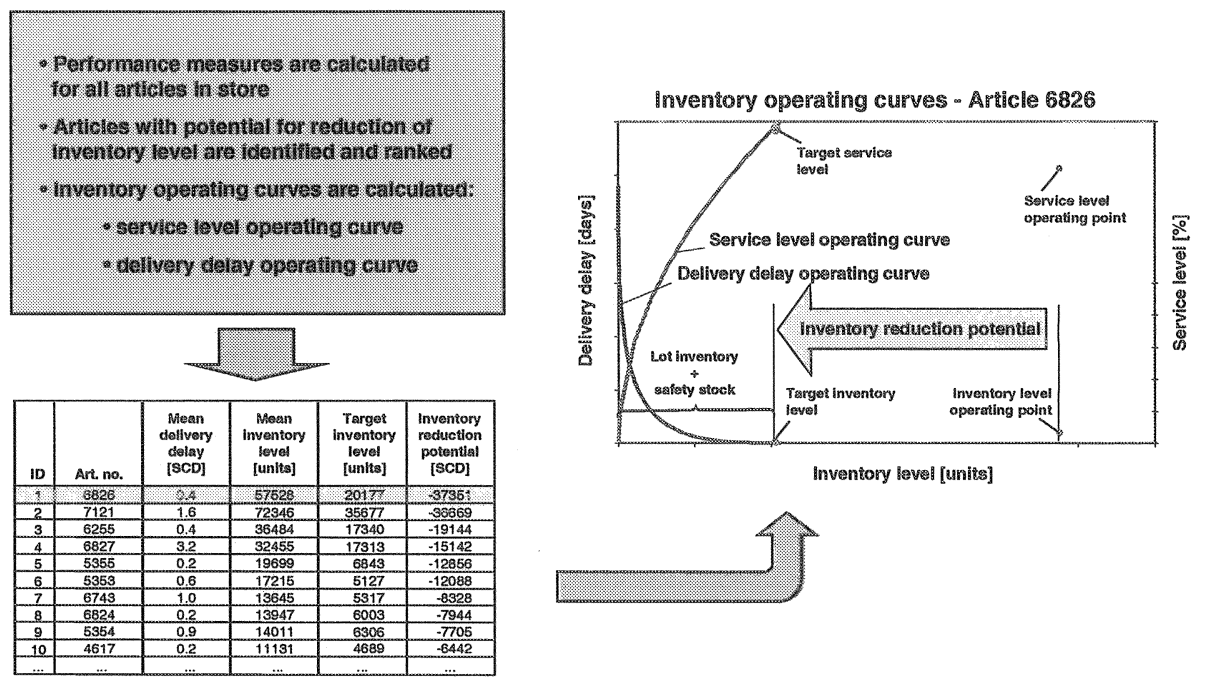

Ranking of inventory reduction potentials

SCD : shop calendar day

Figure 3 - Inventory operating curves reveal potentials for reduction of inventory levels

The shape of the customer service level operating curve is similar to that of a square root function. The service level is zero at the zero inventory level. Above the target inventory level, the service level cannot be increased beyond $100 \%$. The 
delivery delay operating curve has a hyperbolic shape. At the zero inventory level the theoretical delivery delay is infinite, in practice there is a limit to its value. Above the target inventory level, the delivery delay is zero as there is always sufficient inventory in stock to satisfy customer demands. By comparing the actual inventory level with the target inventory level, the potential for inventory reduction can be calculated. For a detailed description of the calculation of inventory operating curves refer to Lutz et al. (2002).

\section{Problems Encountered and Recommendations for Improvement}

The analysis of the final products store found that the inventory levels were not matched to customer demands. For many of the articles held in store, the inventory levels were far too high - resulting in unnecessary capital costs. For a smaller proportion of articles the inventory levels held were too low, thus contributing to an unsatisfactory availability of parts required. The fact that the average customer service level stood at $90 \%$ rather than the aspired $95 \%$ reinforced the impression that the inventory levels were not controlled sufficiently. The reason for this were the shortcomings of the method to determine safety stock levels. IFA proposed a new framework for controlling inventory levels. This is based on a calculation of the safety stock levels required that considers all internal and external factors influencing safety stock requirements. The framework also includes a methodical procedure to continuously monitor and control inventory levels in order to react appropriately to changes in the environment in which the final products store is operated.

\section{Production of Manufacturer}

The production of the manufacturer is planned and controlled according to a production programme based on the customer demand forecasts. Inherently, this means that the production is operated so as to maintain defined inventory levels in the final products store. The monthly planning run translates the forecasts into a production programme that includes manufacturing as well as purchase orders. The manufacturing orders are released into production with a replenishment time of 15 days. IFA used the bottleneck-oriented logistics analysis to investigate the performance of the production department.

\section{Bottleneck-oriented Logistics Analysis}

Bottleneck-oriented logistics analyses evaluate the logistic performance of work systems in a manufacturing department employing three fundamental methodologies: throughput diagrams, logistic operating curves and material flow analyses (see Figure 4) (Penz and Nyhuis, 1995; Nyhuis and Wiendahl, 1999). The major strength of the analysis method is its capability to combine the evaluations of single work systems into an assessment of an entire production system. Once the objectives and boundaries of an investigation have been set, the analysis initially focuses on measuring the logistic performance of single work systems. It primarily considers the performance measures throughput time, due date reliability, work-inprogress (WIP) levels, output rate and resource utilisation (see section on throughput 
diagrams). Taking the actual WIP level as a point of reference, the logistic performance potentials of the work systems are determined (see section on logistic operating curves). The material flow analysis is used to put the logistic performances of the single work systems in relation with each other. By considering the size of the exploitable performance potentials and the contribution to the material flow, the bottleneck work system of the production is identified. This is commonly defined as that work system that has the highest percentage contribution to the throughput time of all orders. In the last phase of the analysis, measures for improvement are developed. These concentrate on the production bottlenecks and other work systems whose performance has a dominant impeding influence on the entire production. The exploitation of their potentials for improvement is the most effective method to improve the overall logistic performance.

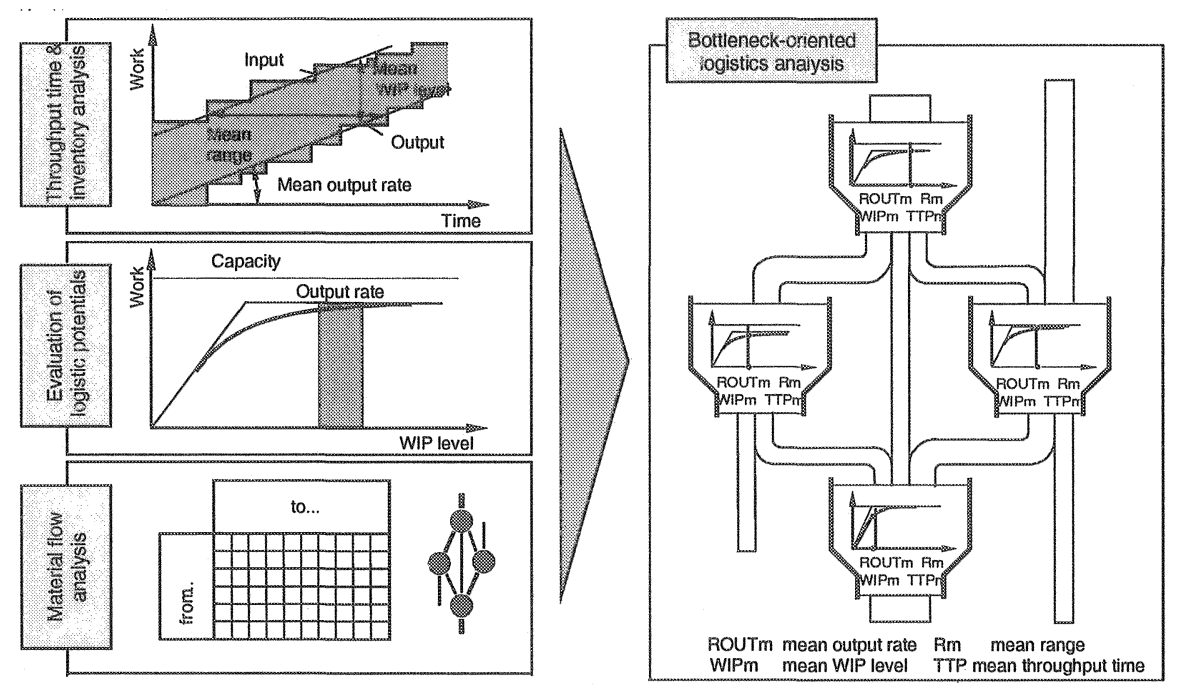

Figure 4 -Methods applied for the bottleneck-oriented logistics analysis

\section{Throughput Diagrams}

Throughput diagrams visualise the dynamic behaviour of the throughput of orders through work systems or stores (Wiendahl, 1995). Figure 5 shows an example of a throughput diagram for a work system. The characteristics of orders are represented in the two dimensions of the diagrams. The input and output dates of orders at the work system are entered on the x-axis, the time axis. The height of an order step entered against the $y$-axis of the diagram depicts the order work content in planned processing hours. The inputs and outputs of separate orders are cumulated over time so that the characteristic step curves appear. The most important performance measures of a work system can be represented in the throughput diagram. The horizontal distance between input and output dates represents the throughput time of an order. The WIP level is equal to the vertical distance between the input and the output curve. The gradient of the input curve is equal to the mean load on the work system, whereas the gradient of the output curve is equal to the mean output rate. Store throughput diagrams are constructed in the very same way. 
Here, the performance measures considered change to inventory level, input rate and output rate.

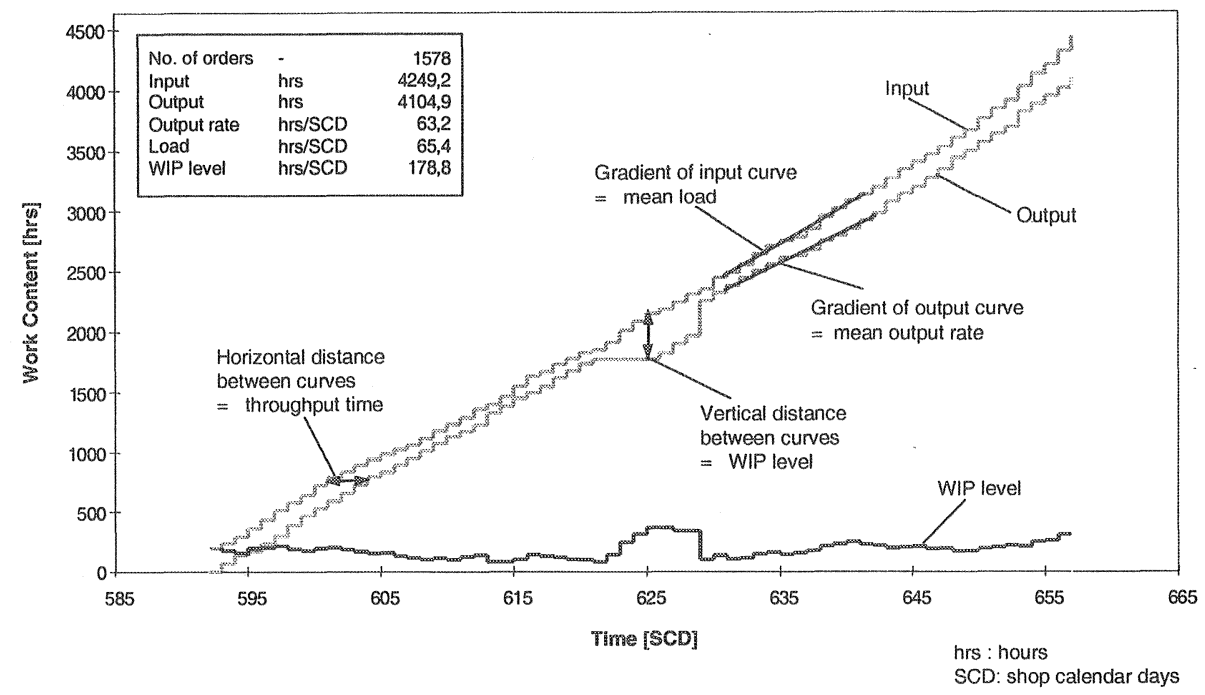

Figure 5 - Throughput diagram of a sample work system

\section{Logistic Operating Curves}

The logistic operating curves approximate the behaviour of logistic performance measures of work systems with variations in WIP levels (see Figure 6) (Nyhuis, 1994; Nyhuis \& Wiendahl, 1999). The curves determine average values of output rate and throughput time for certain WIP levels at a work system. The shape of the operating curves can readily be explained. At first, the output rate rises proportionately to the WIP level. With increasing WIP levels a natural limit of the output rate is reached that is dependent on the capacity of a work system. Further increases in the WIP level only yield negligible increases in the output rate. For low WIP levels, the mean throughput time of orders of a work system remains constant. In this state, neither orders nor work systems have to wait for the availability of another. Only when the WIP level approaches a transient operating state and the number of orders arriving exceeds the number of orders completed, does the throughput time begin to rise proportionately to the WIP level. Similar operating curves can be generated for the utilisation of a work system on the one hand and its range on the other hand. The logistic operating curves can be used for the logistic fine-tuning of work systems that is equivalent to finding a trade-off between different logistic objectives. Mostly, companies aspire to a reduction in throughput time achieved through a reduction of the WIP level as shown in Figure 6. The example also highlights that using the operating curves to specify target WIP levels at all work systems belonging to a process chain can dramatically reduce the throughput time of the entire process - in this particular example by more than $50 \%$. 


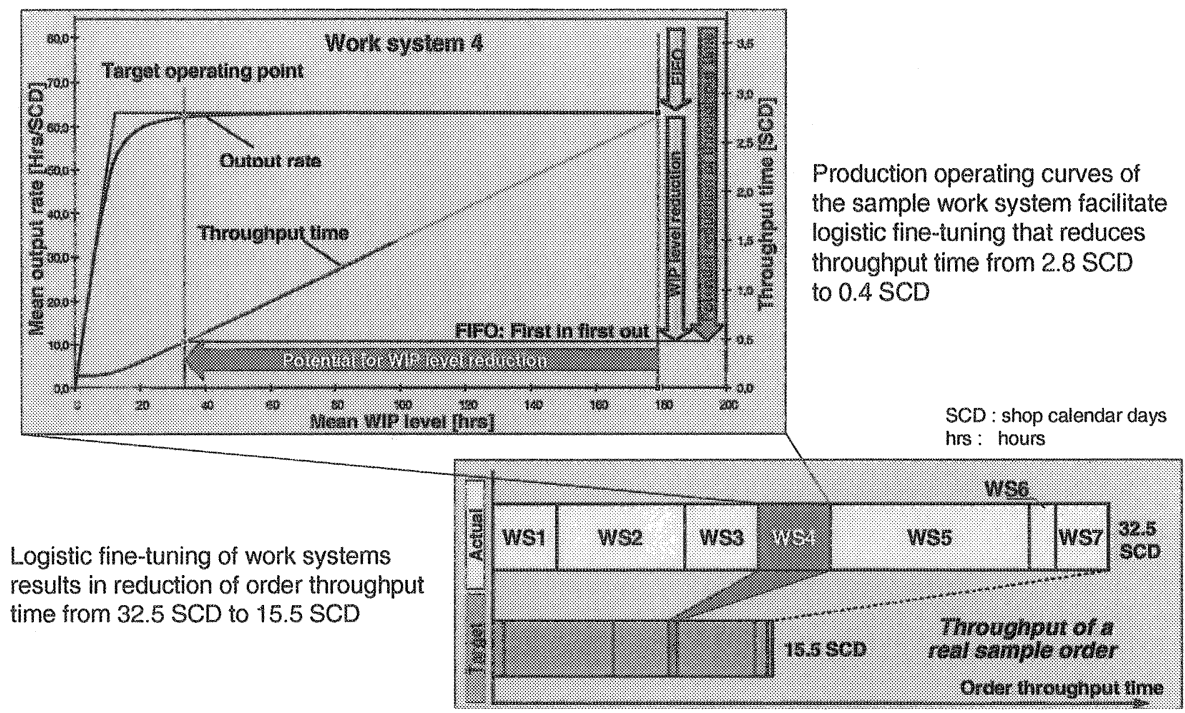

Figure 6 - Logistic operating curves reveal potentials for throughput time reduction

\section{Problems Encountered and Recommendations for Improvement}

The production of the manufacturer exhibited symptoms of logistic process unreliability. The work contents of orders and the throughput times of operations were generally high and subject to wide variations. This led to high levels of positive lateness of manufacturing orders. The average throughput time of orders was equal to 27 shop calendar days (SCD), a value that far exceeds the target replenishment time of 15 SCD. High WIP levels present at most work systems in the production department contributed to the excessive throughput times. Against this setting, IFA suggested a range of measures that sought to balance the load on production and increase the control over WIP levels. The company was advised to reduce WIP levels dramatically by around $40 \%$. Further recommendations concentrated on the management of the throughput of orders. The load on production should be reduced by releasing orders according to the capacity available at the production bottleneck rather than the first work system. Order work contents had to be harmonised and the number of rush orders had to be reduced.

\section{Semi-finished Products Store}

The semi-finished products store was designed as a transit store. The supply chain had intended to deliver the components of the supplier to the store just-in-time (JIT) so that they could proceed to processing in the manufacturer's production within a day of their delivery. The production planning process thus prescribed the delivery due dates and quantities to coincide with concrete material requirements of the production of the manufacturer. IFA examined the semi-finished products store using the logistics-oriented inventory analysis method. The same analysis methods as presented previously were applied. 


\section{Problems Encountered and Recommendations for Improvement}

Analysing the operation of the semi-finished products store and the developments of its average inventory levels over time it became apparent that it was actually operated as a normal inventory-holding store rather than a transit store. This shift of function was caused by external factors. The component availability never reached the requirements set by the intended JIT delivery. To counteract this, safety stock levels had to be specified. This was done in an intuitive rather than a methodical fashion. As a consequence, the actual inventory levels exceeded the required amount by around $76 \%$. Despite this, component unavailability continued to pose problems for the production of the manufacturer. The supply chain had lost control over the inventory levels in this store. IFA recommended changes to the inventory control procedures in order to counteract the problem. Accepting that the semi-finished products store could not immediately be changed into a JIT transit store, a methodical monitoring and controlling procedure for the actual mode of operation was developed. This calculated safety stock levels in a similar fashion as suggested for the final products store. The importance of continually evaluating the relevant performance measures and taking appropriate action was emphasised.

\section{Production of Supplier}

The supplier produces the semi-finished products required by the manufacturer on receipt of a purchase order from the latter. As stated above, the delivery due dates that the supplier has to meet coincide with the date that the components are required by the production of the manufacturer. The target replenishment lead time of the supplier is equal to $15 \mathrm{SCD}$. As for the manufacturer, IFA analysed the production of the supplier by the use of the bottleneck-oriented logistics analysis.

\section{Problems Encountered and Recommendations for Improvement}

The analysis of the single work systems of the production of the supplier did not identify great potentials for improvement of the logistic performance measures. Average WIP levels and throughput times of orders, respectively, exceeded the target values by a relatively low $20 \%$. Nevertheless, the overall logistic performance of the company was unsatisfactory because of organisational shortcomings. The main challenge that faced the supplier was a large backlog of orders. The average backlog was 11 SCD worth of work content. This can be added directly to the average throughput time taking the minimum replenishment lead time to values above $22 \mathrm{SCD}$. In order to counteract low technical process reliabilities the anticipation period between the receipt of an order and the start of production was set at an average of $22 \mathrm{SCD}$. Thus, most orders were released into production after the delivery date requested by the manufacturer. As a result, the delivery reliability of the supplier was only around 5\%. The long set up times imposed on the supplier by technical constraints necessitated large lot sizes. Several purchase orders of the manufacturer were regularly combined in one manufacturing order of the supplier which further impaired the supplier's due date reliability. A lack of meaningful records and feedback data made it very difficult to obtain an overview of the logistic performance of the supplier who was not aware of many of its own shortcomings. 
Countermeasures recommended by IFA aimed at a permanent elimination of the order backlog in order to reduce the replenishment times. The supplier was advised to exploit all possible spare capacities to reduce the backlog and maintain it at near zero levels. Furthermore the anticipation period between receipt of purchase orders and start of production had to be reduced. The supplier was also advised to improve the logistic controlling mechanism by starting to measure, evaluate and control its logistic performance.

\section{RESULTS AND RECOMMENDATIONS}

Once the results of all individual studies were known, these and the suggested measures for improvement had to be consolidated with a view to the requirements of the supply chain as a whole. As the previous section showed, both companies in the supply chain had specific logistic performance deficits. These influence each other and further affect the performance of the supply chain. Shortcomings in the collaboration between the two companies amplified the effects of individual problems. Figure 7 provides an overview of the planning cycle that the supply chain currently uses in order to plan and control the production of its products. The Figure shows the suitability as well as the shortcomings of the procedures applied. Where there existed a deficit, IFA pointed out ways to correct the situation. This section first considers the case of the customised products before treating the standard products, which are distinguished in Figure 7.

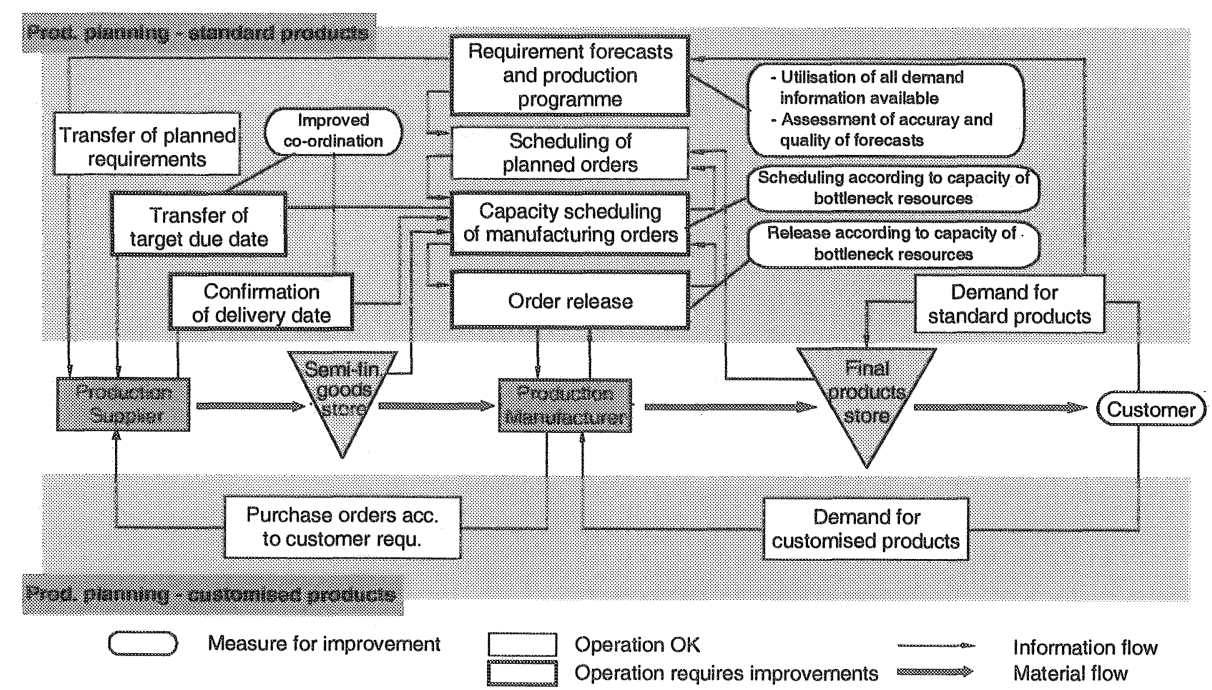

Figure 7 - General problems of the supply chain planning cycle

\section{Problems Encountered - Customised Products}

Figure 7 shows that, conceptually, the current state of the production planning mechanism that is in place for customised products is adequate. For these products, customers place orders with the production of the manufacturer. The manufacturer 
immediately places purchase orders with the supplier that correspond to the requirements of the customer demands. The customer orders have a target lead time of six weeks. This target represents a challenge for the capabilities of the replenishment mechanism. As was seen earlier, the average replenishment time of the supply chain is equal to a combined $60 \mathrm{SCD}$, which is twice the required lead time. Both companies fail to achieve the throughput time figures that would guarantee the desired lead time to their customers. In the case of the manufacturer, the reason for this is the logistic unreliability of the production process. High WIP levels and high order work contents (equivalent to high lot sizes) increase the throughput time of orders. In the case of the supplier, the order backlog as well as the long anticipation period are to blame. These are partly caused by the technical characteristics of the production process. High set up times require high order lot sizes, so that in most cases several purchase orders of the manufacturer are combined into one manufacturing order as was previously pointed out. This not only increased the throughput time of orders that accumulated long queuing times before processing. It also meant that the inventory levels for components of customised products in the semi-finished products store were unnecessarily high due to the larger than expected input lot sizes. The delivery delays meant that this store had to hold safety stock levels to counteract the unavailability of parts. This also applied to the final products store where high inventory levels for customised products buffered the dissatisfactory replenishment time.

\section{Recommendations for Improvement - Customised Products}

Two key demands for improvement arise from the problems pointed out. Firstly, both companies have to radically cut the average throughput time of orders. The manufacturer can achieve this by cutting WIP levels in the production department (Nyhuis, 1994). The supplier should primarily reign in the order backlog and reduce the anticipation period. These measures also positively influence the due date reliability. As a consequence, the inventory levels in the semi-finished and final products stores can be reduced ( $\mathrm{Yu}, 2001)$. Secondly, the lot sizes of the supplier have to be reduced. This is a technical rather than a logistic issue. The process constraints of the supplier required large economic lot sizes. If these constraints can be overcome or simply neglected, smaller, and logistically more suitable, lot sizes can be achieved. Thus, the trade-off is between running smaller, uneconomic lot sizes and achieving the logistic objectives of the supply chain. Smaller lot sizes lead to reduced throughput times. These would, in turn, not only mean an improved responsiveness to customer demands but also yield a substantial reduction in inventory levels both, in the semi-finished and in the final products store.

\section{Problems Encountered - Standard Products}

The problem of not being able to achieve the desired lead times applies to the delivery of standard products as well. In this case, safety stock levels in the final product store had to be even higher in order to make up for the much lower allowable replenishment time (immediate satisfaction of customer orders). The top half of Figure 7 shows several shortcomings of the procedure for processing these parts. Firstly, there was no element of monitoring the forecast accuracy of the 
exponential smoothing forecasting system. As a result, the manufacturer invariably had to manually check the validity of the production programme suggested. Secondly, whereas the rough scheduling of the manufacturing orders included in the production programme was acceptable, the capacity scheduling that ensued failed in several respects. The load orientation of this scheduling run only considered the capacity of the first work system in the material flow of the manufacturer. Thus, the production bottleneck was continually overloaded with orders. Additionally, the due dates that were communicated to the supplier as a result of the capacity scheduling run nearly always had to be dismissed by the supplier. The delivery dates confirmed by the supplier were delayed on the one hand and not considered in the manufacturer's production plans on the other. The results were delivery delays of inputs into the semi-finished products store and increased inventory levels in the store to buffer the delays. Thirdly, the order release mechanism of the manufacturer again ignored the load situation at the production bottlenecks and merely considered the capacity available at the first work system. Thus, the WIP levels in the production of the manufacturer were constantly maintained too high.

Despite sharing a common PPC system, the production planning procedures of the two companies thus disregarded the supply chain partner. Although customer demand data was passed down from the manufacturer to the supplier, usually this was done with a time lag and not immediately after the information became available. The planning process proceeded in separate and iterative steps rather than in a collaborative effort. The companies did not coordinate their schedules. This resulted in mismatches of due dates and the assumption of the wrong values for replenishment times used in drawing up production plans.

\section{Recommendations for Improvement - Standard Products}

Figure 7 presents a range of measures that should be taken in order to improve the supply chain planning process for standard products. The forecasting system should be improved by integrating procedures for assessing the quality of forecasts and taking corrective measures if required. Furthermore, all available information should be utilised to estimate future customer demands even if it is based on experience and does not originate from the formal forecast calculations. Also, the information should be passed to the supplier as soon as possible so that this company is able to anticipate future requirements.

The scheduling mechanisms of the manufacturer should adopt an authentic loadoriented production control that considers the load on the production bottleneck. In this way, the WIP levels are reduced and shorter throughput times can be achieved. The shorter the replenishment times in the supply chain are, the lower the inventory levels in the supply chain can become.

Significant improvements are required of the coordination of due dates between the two supply chain partners. The common PPC system has to be utilised for collaborative planning efforts and the exchange of information that is actually utilised. Important attributes of this exchange of information are completeness, meaning, timing, linkages, transparency and coherence. As a prominent example, the supply chain has to establish an information link between the purchase orders of the manufacturer and the manufacturing orders of the supplier on the one hand, and the 
deliveries to the semi-finished products store by the supplier and the manufacturing orders of the manufacturer on the other hand.

The PPC system should also be used for more intensive production controlling. Performance measures of the supply chain need to be monitored regularly so that the appropriate control activities can be initiated. Generally, both, the production planning and the production control procedures, should take a more holistic view of the supply chain. Local optimisations as in the case of the different lot sizes of supplier and manufacturer lead to detrimental effects for the entire supply chain - in this case unnecessarily high replenishment times and increased inventory levels.

As an alternative to the existing supply chain planning procedures, IFA proposed the combined Kanban-ConWIP control cycle that is shown in Figure 8.

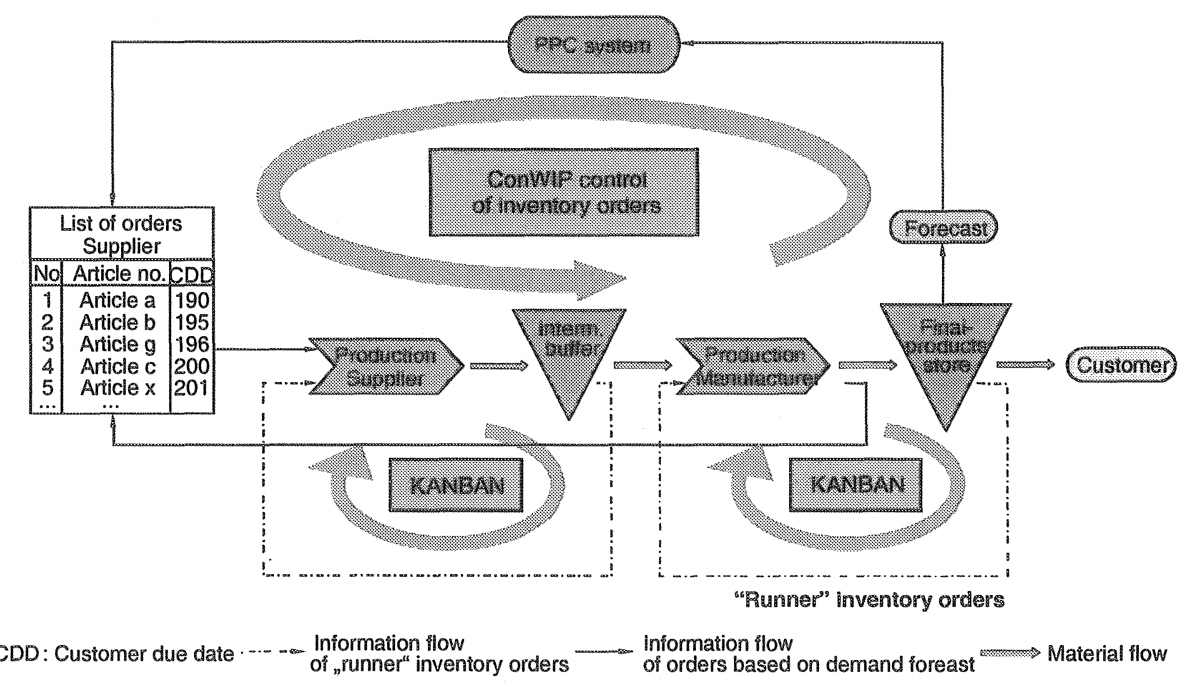

Figure 8-Combined Kanban-CONWIP supply chain planning cycle

So-called "runner" products, for which there is a high as well as stable demand, are controlled by Kanban pull loops. Customers withdraw items from the final products store. Once a defined inventory level is reached a replenishment request triggers the production of the manufacturer. For the processing of the manufacturing order, components are withdrawn from the semi-finished products store. Here, a similar Kanban loop triggers the replenishment by the production of the supplier (see Figure 8). For all other products, IFA specified a ConWIP control procedure (for a detailed description of the ConWIP control procedure see Hopp and Spearman (1996). This utilises a backlog list of orders that controls their release into the production of the supplier. The backlog list is based on accurate customer demand forecasts that are directly transferred to the supplier. On completion of the orders at the supplier the semi-finished products are transferred to the intermediate buffer. All orders completed by the supplier enter a similar backlog list that applies to the manufacturer. One of the advantages of the suggested mechanism is its strict inventory and WIP level control that limits inventory holding costs and ensures short replenishment times. Furthermore, the activities of the two supply chain partners are 
better coordinated. The throughput of orders through the entire supply chain is considered in one planning run rather than having to consolidate separate planning activities of different supply chain partners. The transparency of the planning process is much increased which should enable the supply chain to maintain a better overview over its logistic objectives and performance.

\section{CONCLUSIONS}

Besides the specific results of the investigations which have been covered in detail above, more general conclusions on recommendable supply chain practices can be drawn following the analysis of this particular supply chain.

In terms of the management of the logistic performance the same logic applies for supply chains as for single companies: a trade-off has to be reached between the logistic performance objectives. Single manufacturing companies have to coordinate the order throughput through a network of work systems. Supply chains have to take the coordination effort to a higher level and align the activities of several companies. Thus, a concerted collaborative production planning and control effort by all partners is even more important in a supply chain than in single companies. For companies, the logistic objectives pursued are set by the operations strategy and should therefore coincide. In a supply chain, however, different companies might pursue different strategies and logistic objectives so that conflicts between the partners can arise. In this context, it is important that the supply chain partners reach an agreement as to how their different objectives might be balanced or which objectives should be prioritised. The objective to achieve maximum customer satisfaction could be a guiding principle in finding resolutions of these conflicts.

Conflicting logistic objectives are a reason why modelling the logistic behaviour of a combination of work systems attains such a high level of importance. The logistic process operating curves developed by Wiendahl and Schneider (2001) model the logistic behaviour of a series, or a network, of work systems that operate in combination. For the modelling of this "process" the same logistic performance measures as for the logistic operating curves are considered. In the future, the application of the process operating curves to supply chains will support a holistic view of logistic objectives and offer a more effective tool to resolve conflicts between contradicting objectives.

The possible conflicts of logistic objectives of different supply chain partners also highlight the dangers of a local optimisation of supply chain processes. Solutions that are perfectly suitable to one particular area of the supply chain might severely affect the performance of the entire chain. For this reason, common objectives need to be identified that override the individual interests of single supply chain partners.

IFA discovered a severe lack of formal monitoring and controlling procedures in the supply chain that was the subject of its investigations. The unsatisfactory results of the intuitive approach lead to a further conclusion. Logistic and other performance measures have to be regularly measured, monitored and controlled. For an overview of the performance of a company or supply chain, it is vitally important to measure the correct performance indicators, monitor their trends over time and 
take corrective action if the performance levels achieved are inadequate. Only thus can a process of continuous improvement be initiated.

As a last remark one can conclude that, despite the fact that the supply chain that is subject of this paper might seem a simple case compared to the intricate arrangements covered in literature, the problems encountered are representative of those experienced in other, seemingly more complex, supply chains. The degree of complexity will rise, the nature of the problems will remain the same.

\section{REFERENCES}

[1] Hieber, R.; Alard, R. (2000): Supply Chain Management - A Survey of Practices and Trends in Swiss Industry. In: Strandhagen, J.O.; Alfnes, E. (Eds.): Information and Communication technology (ICT) in Logistics and Production Management. Proceedings of the Annual IFIP WG 5.7 Working Conference, Conference Proceedings 2000. Tromsö, Norway, 28-30 June 2000.

[2] Hopp, W. J.; Spearman, M. L. (1996): Factory Physics - Foundations of Manufacturing Management. Irwin, Chicago.

[3] Lutz, S.; Lödding, H.; Wiendahl, H.-P. (2002): Logistics-oriented Inventory Analysis. In: International Journal of Production Economics (2002), publication pending.

[4] Nyhuis, P. (1994): Logistic Operating Curves - a Comprehensive Method of Rating Logistic Potentials. In: Proceedings of EURO XIII / OR36, University of Strathclyde, Glasgow, 19-22 July 1994, pp. 1 - 24.

[5] Nyhuis, P.; Wiendahl, H.-P. (1999): Logistische Kennlinien. Grundlagen, Werkzeuge und Anwendungen. Springer, Berlin, Heidelberg, New York.

[6] Penz, T.; Nyhuis, P. (1995): Bottleneck-oriented Logistics Analysis as a Basis for Business Re-engineering. In: Browne, J.; O'Sullivan, D. (eds.): Re-engineering the Enterprise - Proceedings of the IFIP WG5.7 Working Conference, Galway, Ireland, 1995, Chapman \& Hall, London et al., pp. 199 - 208.

[7] Schneider, M.; Wiendahl, H.-P. (2002): Modelling and Controlling the Logistic Performance of Manufacturing Departments by the Use of Logistic Process Operating Curves (LPOCs). In: Lee, K. I. (ed.): Proceedings of the 35th CIRP International Seminar on Manufacturing Systems, Seoul, Korea, 13-15 May 2002.

[8] Schönsleben, P. (2000): Integral Logistics Management - Planning and Control of Comprehensive Business Processes. St. Lucie Press, Boca Raton et al.

[9] Wiendahl, H.-P. (1995): Load-oriented Manufacturing Control. Springer, Berlin, Heidelberg, New York.

[10] Wiendahl, H.-P.; Hoebig, M.; Kuhn, A.; Kloth, M.; Weber, J.; Franken, M. (1998): Kennzahlengestützte Prozesse im Supply Chain Management. In: Industrie Management, Vol. 14 (1998), No. 6, pp. 18 - 24.

[11] Yu, K.-W. (2001): Terminkennlinie - Eine Beschreibungsmethodik für die Terminabweichung im Produktionsbereich. Fortschritt-Berichte VDI, Reihe 2, Nr. 576, VDI-Verlag, Düsseldorf. 